\title{
Attitude Stabilization of A Rigid Spacecraft Using Two Control Torques: A Nonlinear Control Approach Based on The Spacecraft Attitude Dynamics*
}

\author{
HARIHARAN KRISHNAN, $\dagger$ MAHMUT REYHANOGLU \\ and HARRIS MCCLAMROCH§
}

Key Words-Spacecraft stabilization; attitude control; discontinuous feedback.

\begin{abstract}
The attitude stabilization problem of a rigid spacecraft using control torques supplied by gas jet actuators about only two of its principal axes is considered. If the uncontrolled principal axis of the spacecraft is not an axis of symmetry, then the complete spacecraft dynamics are small time locally controllable. However, the spacecraft cannot be asymptotically stabilized to any equilibrium attitude using time-invariant continuous feedback. A discontinuous stabilizing feedback control strategy is constructed which stabilizes the spacecraft to any equilibrium attitude. If the uncontrolled principal axis of the spacecraft is an axis of symmetry, the complete spacecraft dynamics are not even assessible. However, the spacecraft dynamics are strongly accessible and small time locally controllable in a reduced sense. The reduced spacecraft dynamics cannot be asymptotically stabilized to any equilibrium attitude using time-invariant continuous feedback, but again a discontinuous stabilizing feedback control strategy is constructed. In both cases, the discontinuous feedback controllers are constructed by switching between several feedback functions which are selected to accomplish a sequence of spacecraft maneuvers. The results of the paper show that although standard nonlinear control techniques are not applicable, it is possible to construct a nonlinear discontinuous control law based on the dynamics of the particular physical system.
\end{abstract}

\section{Introduction}

THE ATTITUDE stabilization problem of a rigid spacecraft using control torques supplied by gas jet actuators about only two of its principal axes is revisited. A rigid spacecraft in general is controlled by three independent actuators and it is well known that three gas jet actuators can be used to accomplish arbitrary reorientation maneuvers of the spacecraft using smooth feedback. The situation considered in this paper may arise due to the failure of one of the actuators of the spacecraft. Since we are considering a

* Received 8 May 1992; revised 3 May 1993; received in final form $30 \mathrm{July,}$ 1993. This paper was not presented at any IFAC meeting. This paper was recommended for publication in revised form by Associate Editor $\mathrm{H}$. Khalil under the direction of Editor Tamer Başar. Corresponding author Professor H. McClamroch. Tel. +1 313764 3310; Fax +1 3137630578 .

† Department of Mechanical and Production Engineering, National University of Singapore, Singapore.

¥Department of Mechanical Engineering, King Fahd University of Petroleum and Minerals, Dhahran, Saudi Arabia.

$\$$ Department of Aerospace Engineering, University of Michigan, Ann Arbor, MI 48109-2140, U.S.A. space-based system, the problem considered here, namely, the attitude stabilization of a spacecraft operating in an actuator failure mode, is an important control problem. The translational and rotational motions of the spacecraft are decoupled and therefore we assume that the center of mass of the spacecraft is not translating.

We first consider the case where the uncontrolled principal axis of the spacecraft is not an axis of symmetry. In this case, the complete spacecraft dynamics are small time locally controllable at any equilibrium attitude. However the spacecraft cannot be asymptotically stabilized to any equilibrium attitude using a time-invariant continuous feedback control law. Using local controllability results, an algorithm which locally asymptotically stabilizes the spacecraft to an isolated equilibrium is proposed in Crouch (1984). That algorithm is extremely complicated and is based on Lie algebraic methods in Hermes (1980). The algorithm yields a piecewise constant discontinuous control. Although very complicated, the algorithm is the only one proposed in the literature thus far which locally asymptotically stabilizes the spacecraft attitude to an equilibrium. In this paper a new discontinuous stabilizing feedback control strategy is constructed which stabilizes the spacecraft to an equilibrium attitude. The control strategy is simple and is based on physical considerations of the problem. We next consider the case where the uncontrolled principal axis of the spacecraft is an axis of symmetry. In this case, the complete spacecraft dynamics are not even accessible. Under some rather weak assumptions, the spacecraft dynamic equations are strongly accessible and small time locally controllable at any equilibrium attitude in a reduced sense. The reduced spacecraft dynamics cannot be asymptotically stabilized to any equilibrium attitude using time-invariant continuous feedback. Nevertheless, a discontinuous feedback control strategy is constructed which achieves attitude stabilization of the spacecraft.

\section{Kinematic and dynamic equations}

Let $J=\operatorname{diag}\left(J_{1}, J_{2}, J_{3}\right), J_{i}>0, i=1,2,3$, be the inertia matrix of the spacecraft in a coordinate frame defined by its principal axes. We assume that the control torques $u_{1}^{\prime}$ and $u_{2}^{\prime}$ are applied about axes represented by unit vectors $b_{1}$ and $b_{2}$ respectively and without loss of generality, let $b_{1}=(1,0,0)^{\tau}$ and $b_{2}=(0,1,0)^{T}$. Define

$$
\left[\begin{array}{l}
u_{1} \\
u_{2}
\end{array}\right]=\left[\begin{array}{l}
\frac{u_{1}^{\prime}}{J_{1}} \\
\frac{u_{2}^{\prime}}{J_{2}}
\end{array}\right] .
$$

Suppose $\omega_{1}, \omega_{2}, \omega_{3}$ are the principal axis components of the absolute angular velocity vector $\omega$ of the spacecraft. Then the equations describing the evolution of the angular velocity 
of the spacecraft are given by

$$
\begin{gathered}
\dot{\omega}=a_{1} \omega_{2} \omega_{3}+u_{1}, \\
\dot{\omega}_{2}=a_{2} \omega_{1} \omega_{3}+u_{2}, \\
\dot{\omega}_{3}=a_{3} \omega_{1} \omega_{2},
\end{gathered}
$$

where

$$
a_{1}=\frac{J_{2}-J_{3}}{J_{1}}, \quad a_{2}=\frac{J_{3}-J_{1}}{J_{2}}, \quad a_{3}=\frac{J_{1}-J_{2}}{J_{3}} .
$$

The orientation of a rigid spacecraft can be specified using various parametrizations of the special orthogonal group $S O(3)$. Here we use the $\mathrm{Z}-\mathrm{Y}-\mathrm{X}$ Euler angle convention for parametrizing the orientation of the rigid spacecraft (Greenwood, 1988). The Euler angles $\psi, \theta, \phi$ are limited to the ranges $-\pi<\psi<\pi,-\pi / 2<\theta<\pi / 2,-\pi<\phi<\pi$. Then we have (Greenwood, 1988)

$$
\begin{gathered}
\dot{\phi}=\omega_{1}+\omega_{2} \sin \phi \tan \theta+\omega_{3} \cos \phi \tan \theta, \\
\dot{\theta}=\omega_{2} \cos \phi-\omega_{3} \sin \phi, \\
\dot{\psi}=\omega_{2} \sin \phi \sec \theta+\omega_{3} \cos \phi \sec \theta
\end{gathered}
$$

\section{Controllability and stabilizability properties}

It is easily verified that the linearization of equations (1)-(6) about any equilibrium has an uncontrollable eigenvalue at the origin. This implies that an inherently nonlinear analysis is necessary in order to characterize the controllability and stabilizability properties of the complete spacecraft dynamics. Moreover, a linear feedback control law cannot be used to asymptotically stabilize the spacecraft to any equilibrium attitude. We now present fundamental results on the controllability and stabilizability properties of the complete spacecraft dynamics described by equations (1)-(6).

Theorem 3.1. The complete spacecraft dynamics described by state equations (1)-(6) are strongly accessible if and only if $J_{1} \neq J_{2}$, i.e. the uncontrolled principal axis is not an axis of symmetry.

Theorem 3.2. The complete spacecraft dynamics described by state equations (1)-(6) are small time locally controllable at any equilibrium if and only if $J_{1} \neq J_{2}$.

Theorem 3.3. The complete spacecraft dynamics described by state equations (1)-(6) cannot be asymptotically stabilized to any equilibrium using a time-invariant continuous feedback control law, but can be asymptotically stabilized to any equilibrium using a piecewise continuous feedback control law.

The reader is referred to Krishnan et al., (1992a) for the proofs of Theorems 3.1 and 3.2. A weaker version of the first part of Theorem 3.3 (with "continuous" replaced by " $C^{1, ")}$ was proved in Byrnes and Isidori (1991). However, the first part of Theorem 3.3 follows from Byrnes and Isidori (1991) using results in Sontag (1989) and Zabczyk (1989). The second part of Theorem 3.3 is a consequence of small time local controllability (Sussman, 1987). Clearly, traditional nonlinear control design methods based on linearization, Lyapunov methods, center manifold theory, or zero dynamics cannot be used to asymptotically stabilize the spacecraft to any equilibrium attitude since there is no general procedure for the design of a discontinuous feedback control.

Although the full set of equations (1)-(6) cannot be asymptotically stabilized to any equilibrium via continuous feedback, one may still wish to design a smooth control law which stabilizes at least a particular subset of state variables. Consider the state equations for $\omega_{1}, \omega_{2}, \omega_{3}, \phi$ and $\theta$ given by equations (1)-(5). These equations are not affected by the Euler angle $\psi$. Asymptotic stabilization of this subset of the original equations corresponds to stabilization of the motion of the spacecraft about an attractor, which is not an isolated equilibrium. A result from Byrnes and Isidori (1991) shows that the closed-loop trajectories can be asymptotically stabilized to the manifold

$$
\Omega=\left\{\left(\omega_{1}, \omega_{2}, \omega_{3}, \phi, \theta, \psi\right): \omega_{1}=\omega_{2}=\omega_{3}=\phi=\theta=0\right\},
$$

using smooth $C^{1}$ feedback.

We mention that although the complete spacecraft dynamics described by equations (1)-(6) cannot be asymptotically stabilized to an equilibrium by continuous feedback, an algorithm generating a piecewise constan discontinuous control has been developed in Crouch (1984) which locally asymptotically stabilizes the complete spacecraft dynamics to an equilibrium. The algorithm requires that $J_{1} \neq J_{2}$, i.e. the uncontrolled principal axis must not be an axis of symmetry. The algorithm is based on Lie algebraic methods in Hermes (1980). The algorithm is extremely complicated and is not an easily implementable control strategy. However, stabilization of the complete spacecraft dynamic equations (1)-(6) is an inherently difficult problem and the algorithm in Crouch (1984) is the only control strategy proposed in the literature thus far.

4. Attitude stabilization of a non-axially symmetric spacecraft

In this section, we assume that the uncontrolled principal axis of the spacecraft is not an axis of symmetry, i.e. $J_{1} \neq J_{2}$, and we restrict our study to the class of discontinuous feedback controllers in order to asymptotically stabilize the complete spacecraft dynamics. Here we present a particular discontinuous feedback strategy, which is obtained by requiring that the spacecraft undergo a sequence of specified maneuvers. Without loss of generality, we assume that the equilibrium attitude to be stabilized is the origin. We first present a physical interpretation of the sequence of maneuvers that transfers any initial state to the origin.

Maneuvers 1-3. Transfer the initial state of the spacecraft to an equilibrium state in finite time; i.e. bring the spacecraft to rest.

There are control laws based on center manifold theory (Aeyels, 1984) and zero dynamics theory (Byrnes and Isidori, 1991) which accomplish this in an asymptotic sense. Here we use a sequence of three maneuvers, and corresponding feedback control laws, which bring the spacecraft to rest in finite time.

Maneuver 4 . Transfer the resulting state to an equilibrium state where $\phi=0$ in finite time; i.e. so that the spacecraft is at rest with $\phi=0$. This maneuver is accomplished using the control torque $u_{1}$ only.

Maneuver 5 . Transfer the resulting state to an equilibrium state where $\phi=0, \theta=0$ in finite time; i.e. so that the spacecraft is at rest with $\phi=0, \theta=0$. This maneuver is accomplished using the control torque $u_{2}$ only.

In order to complete specification of the sequence of maneuvers, the Euler angle $\psi$ must be brought to zero. This cannot be accomplished directly since a control torque cannot be applied about the third principal axis of the spacecraft. However, the resulting state can be transferred to the origin indirectly using three maneuvers. The three maneuvers correspond to three consecutive rotations about the two controlled principal axes of the spacecraft, the first and the third being around the first principal axis. This produces a net change in the orientation of the spacecraft [see Fig. 9 in Marsden et al. (1991)] so that the state of the spacecraft is transferred to the origin in finite time. The three maneuvers are described as follows.

Maneuver 6. Transfer the resulting state to an equilibrium state where $\phi=\pi / 2, \theta=0$ in finite time; i.e. so that the spacecraft is at rest with $\phi=\pi / 2, \theta=0$. This maneuver is accomplished using the control torque $u_{1}$ only.

Maneuver 7 . Transfer the resulting state to the equilibrium state $(0,0,0, \pi / 2,0,0)^{T}$ in finite time. This maneuver is accomplished using the control torque $u_{2}$ only.

Maneuver 8. Transfer the equilibrium state $(0,0,0, \pi / 2$, $0,0)^{T}$ to the equilibrium state $(0,0,0,0,0,0)^{T}$ in finite time. This maneuver is accomplished using the control torque $u_{1}$ only.

Note that, excluding the first three maneuvers where the spacecraft is brought to rest, all subsequent maneuvers are such that the angular velocity component $\omega_{3}$ is maintained 
identically zero. This is accomplished by carrying out maneuvers which require use of only a single control torque at a time. It is convenient to introduce some notation. Throughout, assume $k>0$, and define

$$
G\left(x_{1}, x_{2}\right)= \begin{cases}k & \text { if }\left\{x_{1}+\frac{x_{2}\left|x_{2}\right|}{2 k}>0\right\} \\ & \text { or }\left\{x_{1}+\frac{x_{2}\left|x_{2}\right|}{2 k}=0 \text { and } x_{2}>0\right\} \\ -k & \text { if }\left\{x_{1}+\frac{x_{2}\left|x_{2}\right|}{2 k}<0\right\} \\ & \text { or }\left\{x_{1}+\frac{x_{2}\left|x_{2}\right|}{2 k}=0 \text { and } x_{2}<0\right\} \\ 0 & \text { if }\left\{x_{1}=0 \text { and } x_{2}=0\right\} .\end{cases}
$$

We use the well-known property that the feedback control

$$
u=-G\left(x_{1}-\bar{x}_{1}, x_{2}\right)
$$

for the system

$$
\begin{aligned}
& \dot{x}_{1}=x_{2} \\
& \dot{x}_{2}=u
\end{aligned}
$$

transfers any initial state to the final state $\left(\bar{x}_{1}, 0\right)$ in a finite time. We also use the standard notation that

$$
\operatorname{sign}\left(x_{1}\right)= \begin{cases}1 & \text { if } x_{1}>0 \\ -1 & \text { if } x_{1}<0 \\ 0 & \text { if } x_{1}=0\end{cases}
$$

Our mathematical construction of a control strategy which transfers an arbitrary initial state of the spacecraft to the origin is based on a sequence of equilibrium subsets and a sequence of control functions which transfers a state in one subset to another. Consider the following equilibrium subsets

$$
\begin{aligned}
& M_{1}=\left\{x=(0,0,0, \phi, \theta, \psi)^{T} \mid \phi, \theta, \psi \text { arbitrary }\right\}, \\
& M_{2}=\left\{x=(0,0,0,0, \theta, \psi)^{T} \mid \theta, \psi \text { arbitrary }\right\}, \\
& M_{3}=\left\{x=(0,0,0,0,0, \psi)^{T} \mid \psi \text { arbitrary }\right\}, \\
& M_{4}=\left\{x=\left(0,0,0, \frac{\pi}{2}, 0, \psi\right)^{T} \mid \psi \text { arbitrary }\right\}
\end{aligned}
$$

We now present the feedback control laws that accomplish the sequential maneuvers described above; for each case we show that a desired terminal state which defines the maneuver is reached.

Transferring any initial state to a state in $M_{1}$

In order to transfer the arbitrary initial state to a final state which satisfies $\omega_{1}=\omega_{2}=\omega_{3}=0$ three sequential maneuvers are required. The first maneuver results in $\omega_{1}=\omega_{2}=0$ while $\omega_{3} \neq 0$ in general; the second maneuver results in $\omega_{1}=\omega_{i}^{*}$ and $\omega_{2}=\omega_{2}^{*}$, where $\omega_{1}^{*}, \omega_{2}^{*}$ are chosen to guarantee that at the end of the third maneuver $\omega_{1}=\omega_{2}=\omega_{3}=0$. These three maneuvers are described in detail as follows.

Maneuver 1. Let $\left(\omega_{1}^{0}, \omega_{2}^{0}, \omega_{3}^{0}, \phi^{0}, \theta^{0}, \psi^{0}\right)^{T}$ denote an initial state for the complete spacecraft dynamics described by equations (1)-(6). Define

$$
\begin{aligned}
& v_{1}=a_{1} \omega_{2} \omega_{3}+u_{1}, \\
& v_{2}=a_{2} \omega_{3} \omega_{1}+u_{2} .
\end{aligned}
$$

Equations (1)-(3) can now be rewritten as

$$
\begin{gathered}
\dot{\omega}_{1}=v_{1}, \\
\dot{\omega}_{2}=v_{2}, \\
\dot{\omega}_{3}=a_{3} \omega_{1} \omega_{2} .
\end{gathered}
$$

Apply the feedback control functions

$$
\begin{aligned}
& v_{1}=-k \operatorname{sign} \omega_{1}, \\
& v_{2}=-k \operatorname{sign} \omega_{2} .
\end{aligned}
$$

It is easy to see that after a finite time given by $\max \left(\left|\omega_{1}^{0}\right| / k\right.$, $\left.\left|\omega_{2}^{0}\right| / k\right), \omega_{1}=\omega_{2}=0$; at this instant let $\omega_{3}=\bar{\omega}_{3}$ where the constant value $\bar{\omega}_{3}$ can be evaluated.

Maneuver 2. Apply the feedback control functions

$$
\begin{aligned}
& v_{1}=-k \operatorname{sign}\left(\omega_{1}-\omega_{1}^{*}\right), \\
& v_{2}=-k \operatorname{sign}\left(\omega_{2}-\omega_{2}^{*}\right),
\end{aligned}
$$

where

$$
\omega_{1}^{*}=\left(\frac{3 k\left|\bar{\omega}_{3}\right|}{2\left|a_{3}\right|}\right)^{\frac{1}{2}}, \quad \omega_{2}^{*}=-\omega_{1}^{*} \operatorname{sign} \bar{\omega}_{3} \operatorname{sign} a_{3} .
$$

It is again easy to see that after a finite time given by $\omega_{i}^{*} / k$, $\omega_{1}=\omega_{1}^{*}, \omega_{2}=\omega_{2}^{*}$, and in addition it can be shown that $\omega_{3}=\bar{\omega}_{3} / 2$.

Maneuver 3. Apply the feedback control functions

$$
\begin{aligned}
& v_{1}=-k \operatorname{sign} \omega_{1}, \\
& v_{2}=-k \operatorname{sign} \omega_{2} .
\end{aligned}
$$

It can be seen that after a finite time given by $\omega_{1}^{*} / k, \omega_{1}=0$, $\omega_{2}=0$ and it can be shown that $\omega_{3}=0$.

Consequently, the resulting state after these three sequential maneuvers is $\left(0,0,0, \phi^{1}, \theta^{1}, \psi^{1}\right)^{T} \in M_{1}$ for some $\phi^{1}, \theta^{1}, \psi^{1}$.

Transferring a state in $M_{1}$ to a state in $M_{2}$

Maneuver 4 . Let $\left(0,0,0, \phi^{1}, \theta^{1}, \psi^{1}\right)^{T}=M_{1}$ denote a state of the spacecraft. Apply the feedback control functions

$$
\begin{aligned}
& u_{1}=-G\left(\phi, \omega_{1}\right), \\
& u_{2}=0 .
\end{aligned}
$$

It follows that $\omega_{2}=0, \omega_{3}=0, \theta=\theta^{1}, \psi=\psi^{1}$, satisfy equations (2), (3), (5), (6) while equations (1), (4) become

$$
\begin{aligned}
\dot{\omega}_{1} & =-G\left(\phi, \omega_{1}\right), \\
\dot{\phi} & =\omega_{1} .
\end{aligned}
$$

Consequently, after a finite time $\omega_{1}=0, \phi=0$; and thus the maneuver transfers a state $\left(0,0,0, \phi^{1}, \theta^{1}, \psi^{1}\right)^{T} \in M_{1}$ to the state $\left(0,0,0,0, \theta^{1}, \psi^{1}\right)^{T} \in M_{2}$ in finite time.

Transferring a state in $M_{2}$ to a state in $M_{3}$

Maneuver 5. Let $\left(0,0,0,0, \theta^{1}, \psi^{\prime}\right)^{T} \in M_{2}$ denote a state of the spacecraft. Apply the feedback control functions

$$
\begin{aligned}
& u_{1}=0, \\
& u_{2}=-G\left(\theta, \omega_{2}\right) .
\end{aligned}
$$

It follows that $\omega_{1}=0, \omega_{3}=0, \phi=0, \psi=\psi^{1}$, satisfy equations (1), (3), (4), (6) while equations (2), (5) become

$$
\begin{aligned}
\dot{\omega}_{2} & =-G\left(\theta, \omega_{2}\right), \\
\dot{\theta} & =\omega_{2} .
\end{aligned}
$$

Consequently, after a finite time $\omega_{2}=0, \theta=0$; and thus the maneuver transfers a state $\left(0,0,0,0, \theta^{1}, \psi^{1}\right)^{T} \in M_{2}$ to the state $\left(0,0,0,0,0, \psi^{1}\right)^{T} \in M_{3}$ in finite time.

Transferring a state in $M_{3}$ to a state in $M_{4}$

Maneuver 6 . Let $\left(0,0,0,0,0, \psi^{\prime}\right)^{T} \in M_{3}$ denote a state of the spacecraft. Apply the feedback control functions

$$
\begin{aligned}
& u_{1}=-G\left(\phi-\frac{\pi}{2}, \omega_{1}\right), \\
& u_{2}=0 .
\end{aligned}
$$

It follows that $\omega_{2}=0, \omega_{3}=0, \theta=0, \psi=\psi^{\prime}$, satisfy equations (2), (3), (5), (6) while equations (1), (4) become

$$
\begin{aligned}
\dot{\omega}_{1} & =-G\left(\phi-\frac{\pi}{2}, \omega_{1}\right), \\
\dot{\phi} & =\omega_{1} .
\end{aligned}
$$

Consequently, after a finite time $\omega_{1}=0, \phi=\pi / 2$; and thus the maneuver transfers a state $\left(0,0,0,0,0, \psi^{1}\right)^{T} \in M_{3}$ to the state $\left(0,0,0, \pi / 2,0, \psi^{\prime}\right) \in M_{4}$ in finite time. 
Transferring a state in $M_{4}$ to $(0,0,0, \pi / 2,0,0)^{T}$ Maneuver 7. Let $\left(0,0,0, \pi / 2,0, \psi^{\prime}\right)^{T} \in M_{4}$ denote a state of the spacecraft. Apply the feedback control functions

$$
\begin{aligned}
& u_{1}=0, \\
& u_{2}=-G\left(\psi, \omega_{2}\right) .
\end{aligned}
$$

It follows that $\omega_{1}=0, \omega_{3}=0, \phi=\pi / 2, \theta=0$, satisfy equations (1), (3), (4), (5) while equations (2), (6) become

$$
\begin{aligned}
\dot{\omega}_{2} & =-G\left(\psi, \omega_{2}\right), \\
\dot{\psi} & =\omega_{2} .
\end{aligned}
$$

Consequently, after a finite time $\omega_{2}=0, \psi=0$; and thus the maneuver transfers a state $\left(0,0,0, \pi / 2,0, \psi^{1}\right)^{T} \in M_{4}$ to the state $(0,0,0, \pi / 2,0,0)^{T}$ in finite time.

Transferring $(0,0,0, \pi / 2,0,0)^{T}$ to $(0,0,0,0,0,0)$

Maneuver 8. Let $(0,0,0, \pi / 2,0,0)^{T}$ denote the state of the spacecraft. Apply the feedback control functions

$$
\begin{aligned}
& u_{1}=-G\left(\phi, \omega_{1}\right), \\
& u_{2}=0 .
\end{aligned}
$$

It follows that $\omega_{2}=0, \omega_{3}=0, \theta=0, \psi=0$, satisfy equations (2), (3), (5), (6) while equations (1), (4) become

$$
\begin{aligned}
\dot{\omega}_{1} & =-G\left(\phi, \omega_{1}\right), \\
\dot{\phi} & =\omega_{1} .
\end{aligned}
$$

Consequently, after a finite time $\omega_{1}=0, \phi=0$; and thus the maneuver transfers $(0,0,0, \pi / 2,0,0)^{\frac{T}{t}}$ to the state $(0,0,0,0,0,0)^{r}$ in finite time.

In summary, the feedback control strategy outlined above can be implemented by sequential switching between the following feedback functions.

Maneuver 1. Apply

$$
\begin{aligned}
& u_{1}^{\prime}(x)=-a_{1} \omega_{2} \omega_{3}-k \operatorname{sign} \omega_{1}, \\
& u_{2}^{1}(x)=-a_{2} \omega_{3} \omega_{1}-k \operatorname{sign} \omega_{2},
\end{aligned}
$$

until $\left(\omega_{1}, \omega_{2}, \omega_{3}\right)=\left(0,0, \bar{\omega}_{3}\right)$ for some value $\bar{\omega}_{3}$; then go to Manuever 2.

Maneuver 2. Compute

$$
\omega_{1}^{*}=\left(\frac{3 k\left|\bar{\omega}_{3}\right|}{2\left|a_{3}\right|}\right)^{1 / 3}, \quad \omega_{2}^{*}=-\left(\frac{3 k\left|\bar{\omega}_{3}\right|}{2\left|a_{3}\right|}\right)^{1 / 3} \operatorname{sign} \bar{\omega}_{3} \operatorname{sign} a_{3} ;
$$

apply

$$
\begin{aligned}
& u_{1}^{2}(x)=-a_{1} \omega_{2} \omega_{3}-k \operatorname{sign}\left(\omega_{1}-\omega_{1}^{*}\right), \\
& u_{2}^{2}(x)=-a_{1} \omega_{3} \omega_{1}-k \operatorname{sign}\left(\omega_{2}-\omega_{2}^{*}\right),
\end{aligned}
$$

until $\left(\omega_{1}, \omega_{2}, \omega_{3}\right)=\left(\omega_{1}^{*}, \omega_{2}^{*}, \bar{\omega}_{3} / 2\right)$; then go to Maneuver 3 . Maneuver 3. Apply

$$
\begin{aligned}
& u_{1}^{3}(x)=-a_{1} \omega_{2} \omega_{3}-k \operatorname{sign} \omega_{1}, \\
& u_{2}^{3}(x)=-a_{2} \omega_{3} \omega_{1}-k \operatorname{sign} \omega_{2},
\end{aligned}
$$

until $\left(\omega_{1}, \omega_{2}, \omega_{3}\right)=(0,0,0)$, i.e. $\left(\omega_{1}, \omega_{2}, \omega_{3}, \phi, \theta, \psi\right)^{T} \epsilon$ $M_{1} ;$ then go to Maneuver 4 .

Maneuver 4. Apply

$$
\begin{aligned}
& u_{1}^{4}(x)=-G\left(\phi, \omega_{1}\right), \\
& u_{2}^{4}(x)=0,
\end{aligned}
$$

until $\left(\omega_{1}, \omega_{2}, \omega_{3}, \phi\right)=(0,0,0,0)$, i.e. $\left(\omega_{1}, \omega_{2}, \omega_{3}, \phi, \theta\right.$, $\psi)^{T} \in M_{2}$; then go to Maneuver 5 .

Maneuver 5. Apply

$$
\begin{aligned}
& u_{1}^{5}(x)=0, \\
& u_{2}^{5}(x)=-G\left(\theta, \omega_{2}\right),
\end{aligned}
$$

until $\left(\omega_{1}, \omega_{2}, \omega_{3}, \phi, \theta\right)=(0,0,0,0,0)$, i.e. $\left(\omega_{1}, \omega_{2}, \omega_{3}, \phi\right.$, $\theta, \psi)^{T} \in M_{3}$; then go to Maneuver 6 .
Maneuver 6. Apply

$$
\begin{aligned}
& u_{1}^{6}(x)=-G\left(\phi-\frac{\pi}{2}, \omega_{1}\right), \\
& u_{2}^{6}(x)=0,
\end{aligned}
$$

until $\left(\omega_{1}, \omega_{2}, \omega_{3}, \phi, \theta\right)=(0,0,0, \pi / 2,0)$, i.e. $\left(\omega_{1}, \omega_{2}, \omega_{3}\right.$, $\phi, \theta, \psi)^{T} \in M_{4}$; then go to Maneuver 7 .

Maneuver 7. Apply

$$
\begin{aligned}
& u_{1}^{7}(x)=0, \\
& u_{2}^{7}(x)=-G\left(\psi, \omega_{2}\right),
\end{aligned}
$$

until $\left(\omega_{1}, \omega_{2}, \omega_{3}, \phi, \theta, \psi\right)=(0,0,0, \pi / 2,0,0)$; then go to Maneuver 8.

Maneuver 8. Apply

$$
\begin{aligned}
& u_{1}^{8}(x)=-G\left(\phi, \omega_{1}\right), \\
& u_{2}^{8}(x)=0,
\end{aligned}
$$

until $\left(\omega_{1}, \omega_{2}, \omega_{3}, \phi, \theta, \psi\right)=(0,0,0,0,0,0)$.

This feedback control strategy achieves attitude stabilization of the spacecraft by executing a sequence of maneuvers. This strategy is discontinuous and nonclassical in nature. Justification that it stabilizes the complete spacecraft dynamics to an equilibrium attitude in finite time, under the ideal model assumptions, follows as a consequence of the construction procedure. A computer implementation of the feedback control strategy can be easily carried out.

\section{Attitude stabilization of an axially symmetric spacecraft}

From the analysis made in Section 3 , we find that the complete dynamics of a spacecraft controlled by two control torques supplied by gas jet actuators fail to be controllable or even accessible if the uncontrolled principal axis is an axis of symmetry of the spacecraft, i.e. if $J_{1}=J_{2}$. In this section we concentrate on the case where the uncontrolled principal axis of the spacecraft is an axis of symmetry, i.e. $J_{1}=J_{2}$. In particular we ask the question: what restricted control and stabilization properties of the spacecraft can be demonstrated in this case? Our analysis begins by demonstrating that, under appropriate restrictions of interest, the spacecraft equations can be expressed in a reduced form. Controllability and stabilizability properties for this case follow from an analysis of the reduced equations.

Consider the equations (1)-(6). If $J_{1}=J_{2}$ and $\omega_{3}(0) \neq 0$, then $\omega_{3}$ cannot be transferred to zero using any control function. If we assume that $\omega_{3}(0)=0$, then $\omega_{3} \equiv 0$. Under the restriction $\omega_{3}(0)=0$, the reduced spacecraft dynamics for this case are described by

$$
\begin{gathered}
\dot{\omega}_{1}=u_{1}, \\
\dot{\omega}_{2}=u_{2}, \\
\dot{\phi}=\omega_{1}+\omega_{2} \sin \phi \tan \theta, \\
\dot{\theta}=\omega_{2} \cos \phi, \\
\dot{\psi}=\omega_{2} \sin \phi \sec \theta .
\end{gathered}
$$

The following results can now be easily shown. The proofs of Theorem 5.1 and Theorem 5.2 are similar to the proofs of Theorem 3.1 and Theorem 3.2 respectively in Krishnan et al. (1992a). Theorem 5.3 follows from the results in Brockett (1983), Sontag (1989), and Zabczyk (1989).

Theorem 5.1. The reduced dynamics of an axially symmetric spacecraft controlled by two pairs of gas jet actuators as described by equations (10)-(14) are strongly accessible.

Theorem 5.2. The reduced dynamics of an axially symmetric spacecraft controlled by two pairs of gas jet actuators as described by equations (10)-(14) are small time locally controllable at any equilibrium.

Theorem 5.3. The reduced dynamics of an axially symmetric spacecraft controlled by two pairs of gas jet actuators as described by equations (10)-(14) cannot be asymptotically 
stabilized to any equilibrium using a time-invariant continuous feedback control law, but can be asymptotically stabilized using a piecewise continuous feedback control law.

The implications of the properties stated above are as follows. For all initial conditions that satisfy $\omega_{3}(0)=0$, the axially symmetric spacecraft controlled by two pairs of gas jet actuators can be controlled to any equilibrium attitude. However, any time-invariant feedback control law that asymptotically stabilizes the spacecraft to any equilibrium attitude must necessarily be discontinuous. Thus arbitrary reorientation of the spacecraft can be achieved if $\omega_{3}(0)=0$; if $\omega_{3}(0) \neq 0$, reorientation of the spacecraft to any equilibrium attitude cannot be achieved. Conveniently, it turns out that sequential execution of the maneuvers defined as Manuevers 3 through 8 in the previous section transfers any initial state of the reduced spacecraft dynamics $(10)-(14)$ to the origin in finite time. The physical interpretation of the manuevers is the same as described previously. The reader is referred to Krishnan et al., (1992b) for additional details. An alternate discontinuous control strategy which achieves attitude stabilization of the spacecraft is presented in Krishnan et al. (1992a).

\section{Conclusion}

The attitude stabilization problem of a spacecraft using control torques supplied by gas jet actuators about only two of its principal axes has been considered. If the uncontrolled principal axis is not an axis of symmetry of the spacecraft, the complete spacecraft dynamics cannot be asymptotically stabilized to any equilibrium attitude using continuous feedback. A discontinuous feedback control strategy has been constructed which stabilizes the spacecraft to any equilibrium attitude in finite time. If the uncontrolled principal axis is an axis of symmetry of the spacecraft, the complete spacecraft dynamics cannot be stabilized. The reduced spacecraft dynamics cannot be asymptotically stabilized using continuous feedback, but again a discontinuous feedback control strategy has been proposed which stabilizes the spacecraft (in the reduced sense) to any equilibrium attitude in finite time. The results of the paper show that although standard nonlinear control techniques do not apply, it is possible to construct a stabilizing control law by performing a sequence of maneuvers.

One of the advantages of the development in this paper is that feedback control strategies are constructed which guarantee attitude stabilization in a finite time. The total time required to complete the spacecraft reorientation is the sum of the times required to complete the sequence of maneuvers described. From the analysis provided, it should be clear that the time required to complete each maneuver depends on the single positive parameter $k$ in the corresponding control law. There is a trade off between the required control levels, determined by the selection of $k$, and the resulting times to complete each of the maneuvers and hence the total time required to reorient the spacrcraft. In particular, the time to reorient the spacecraft from a given initial state to the origin can be expressed as a function of the value of the parameter $k$ and of the initial state.

We have presented a sequence of maneuvers which achieves the desired spacecraft attitude stabilization. There are many other maneuver sequences, and corresponding feedback control strategies, which will also achieve the desired attitude stabilization of the spacecraft. But each such strategy is necessarily discontinuous. We have demonstrated the closed-loop properties for the special feedback control strategies presented. Our analysis was based on a number of assumptions which are required to justify the mathematical models studied. Further robustness analysis is required to determine effects of model uncertainties and external disturbances. Unfortunately, such robustness analysis is quite difficult since the closed-loop vector fields are necessarily discontinuous. Perhaps, feedback control strategies which stabilize the spacecraft attitude, different from ones presented in this paper, would provide improved closed-loop robustness.

Acknowledgement-This work was partially supported by NSF Grant No. MSS-9114630 and by NASA Grant No. NAG-1-1419.

\section{References}

Aeyels, D. (1984). Stabilization of a class of nonlinear systems by a smooth feedback control. Systems and Control Letters, 5, 289-294.

Brockett, R. W. (1983). Asymptotic stability and feedback stabilization. In R. W. Brockett, R. S. Millman, H. J. Sussman, (Eds), Differential Geometric Control Theory, pp. 181-191, Birkhauser, Boston.

Byrnes, C. I. and A. Isidori (1991). On the attitude stabilization of rigid spacecraft. Automatica, 27, 87-95.

Crouch, P. E. (1984). Spacecraft attitude control and stabilization: Applications of geometric control theory to rigid body models. IEEE Trans. Auto. Control, AC-29, 321-331.

Greenwood, D. (1988). Intermediate Dynamics, 2nd ed. Prentice-Hall, Englewood Cliffs, NJ.

Hermes, H. (1980). On the synthesis of a stabilizing feedback control via Lie algebraic methods. SIAM J. Control and Optimization, 18, 352-361.

Krishnan, H., N. H. McClamroch and M. Reyhanoglu (1992a). On the attitude stabilization of a rigid spacecraft using two control torques. Proc. of the American Control Conference, Chicago, Illinois, pp. 1990-1995.

Krishnan, H., M. Reyhanoglu and N. H. McClamroch (1992b). Attitude stabilization of a rigid spacecraft using gas jet actuators operating in a failure mode. Proc. of the IEEE Conference on Decision and Control, Tucson, Arizona, pp. 1612-1617.

Sontag, E. D. (1989). Feedback stabilization on nonlinear systems. Proc. of the MTNS-89, Birkhauser, BaselBoston.

Sontag, E. D. and H. J. Sussman (1988). Further comments on the stabilizability of the angular velocity of a rigid body. Systems and Control Letters, 12, 213-217.

Sussman, H. J. (1987). A general theorem on local controllability. SIAM J. Control and Optimization, 25, 158-194.

Zabczyk, J. (1989). Some comments on stabilizability. Appl. Math. Optim., 19, 1-9. 